on the behaviour of materials at high temperatures in the presence of nuclear fission. There is some information on the effects of radiation on different types of materials at room temperature, however. This information was summarized by Dr. Clifford Weber, of the General Electric Company.

The effects of high pressures on the structure and properties of refractory materials were discussed by both Dean S. S. Kistler, of the University of Utah, and Dr. H. T. Hall, of Brigham Young University. Most startling of their comments was the fact that the melting point of certain refractories has been raised more than 1,000 deg. C. upon application of pressure. On the other hand, with substances that decrease in volume as they melt, the melting point is depressed by pressure. For example, germanium, under pressure, melts at a temperature $600 \mathrm{deg} C$. less than without pressure.

Although the details of the recently publicized successes in the manufacture of diamonds could not be disclosed, Dr. H. T. Hall, who was associated with that work, discussed some of its general aspects. In this he gave a striking example of the power of theoretical examination of high-temperature problems. $\mathrm{H}_{\Theta}$ discussed a calculation demonstrating that known thermodynamic data for graphite and diamond and known kinetic data for conversion of diamond to graphite prove the impossibility of converting graphite to diamond in any temperaturepressure range achievable by conceivable means now possible for the investigation of phenomena that occur at pressures of 200,000 atmospheres and temperatures as high as $500^{\circ} \mathrm{C}$.

Dean Kistler spoke of certain interesting resemblances of boron nitride to graphite. $\mathrm{He}$ directed attention to the fact that, from fundamental considerations, a cubic form of boron nitride with mechanical properties similar to diamond may be expected. The significance of such a possibility is obvious inasmuch as no known material has a hardness between that of boron carbide (2,700 Kroop $\left.\mathrm{K}_{100}\right)$ and diamond $(6,500)$. Dean Kistler held out the hope that a number of completely new materials may be formulated and produced, quite possibly with attractive high-temperature characteristics. As an example he remarked that a new form of silica has been produced simply by compressing quartz above 80,000 atmospheres. Cœsite was also mentioned by Hall as another modification of silica, produced by direct conversion from quartz heated to $2,000^{\circ} \mathrm{C}$. at about 100,000 atmospheres pressure.

Dr. George Duvall and Dr. Milton C. Kells, of Stanford Research Institute, as well as Prof. B. H. Sage, of the California Institute of Technology, mentioned shock waves and high-speed nozzles as useful sources of high-temperature kinetic data. Reactions with half-times as low as five microseconds can be studied in shock tubes. A shock tube can yield a temperature of $11,000^{\circ} \mathrm{K}$. in less than $12 \mu$ sec. High-temperature jets are being actively investigated as commercial sources of gases, such as acetylene.

Progress with the technique of melting and refining metals suspended in space by electromagnetic fields to avoid contamination with container walls was reported by I. E. Campbell, of the Battelle Memorial Institute, and J. C. R. Kelly, of the Westinghouse Electric Corporation. Kelly pointed out that the levitation-melting technique may also be useful for determining the surface tension of molten metals.
The Berkeley meeting made it clear that reactor and jet-engine development will continue to provide a tremendous impetus to research on high-temperature materials, and that high-temperature chemical processing is likely to witness spectacular expansion. It also demonstrated the comparative paucity of information in the high-temperature field. This was underlined by an example given by Kistler. $\mathrm{He}$ pointed out that, although silicon carbide is one of the oldest and best known synthetic high-temperature products, we still do not understand such things as the effects of furnace atmospheres on the rate of its formation, the factors determining whether the cubic or hexagonal form of the compound will be produced, or the effects of impurities on such important properties as conductivity, oxidation resistance, and grinding characteristics. Clearly, much remains to be done.

The papers given by the members of the various panels, with answers to the many questions put from the floor, are being printed and are expected to be issued about October 30; copies will be available from the Public Relations Department, Stanford Research Institute, Menlo Park, California (price 5 dollars each).

\section{CHROMOSOME PROBLEMS}

A

CONFERENCE on chromosomes was held in 1 the Landbouwhogeschool, Wageningen, Holland, during April 16-19, Profs. H. J. C. Tendeloo and R. Prakken being chairman and secretary respectively. The programme consisted of seven lectures with discussions.

Prof. E. Heitz (Tübingen) spoke first on chromosome structure in the nucleus, during mitosis and meiosis.

There are five different types of structural elements recognized in a chromosome: the spiral, the chromomeres, the centromere (primary constriction, kinetochore), the secondary constrictions (SAT-zones) and the heterochromatine. Four of these are directly (heterochromatin) or indirectly (the spiral, the centromere, the SAT-zones) demonstrable in the nucleus. These serve as proof of the individuality of the chromosomes within the nucleus.

The essential element of every chromosome, the spiral, which is tightly coiled during metaphase and anaphase, uncoils during telophase and gives rise, after the calymma has disappeared, to the threadlike structure of the nucleus. This structure has been observed in the living state and can also be demonstrated in the nucleus after fixation.

Often, certain parts of chromosomes do not uncoil during telophase and probably do not lose their calymmatic substance. Since such heterochromatic pieces occupy defined positions within the chromosomes, they can be recognized as more or less compact and highly coloured pieces in their respective positions.

Since rather large pieces of the chromosome on both sides of the centromere are often heterochromatic, the position of the centromere can be demonstrated in the proximal part of the young nucleus.

Chromomeres themselves (that is, compact, small and nucleal positive pieces within the anucleal uncoiled zygotene or pachytene chromosome-fibres seen during meiosis) cannot be demonstrated in the somatic nucleus, although heterochromatic pieces may be mistaken for them. 
Secondary constrictions can be recognized in the nuclei by means of their function. They condense the nucleoli during telophase. Since the number and position of every secondary constriction are definite, the number and position of the nucleoli, in the young nuclei at least, correspond to the number and position of the secondary constrictions.

There are many indications that the major spiral contains a minor spiral. Furthermore, there are many reasons to suspect the existence of a third, perhaps molecular, spiral, running in the minor spiral, for example, the enormous length of the fibrils in the giant Dipteran chromosomes may thus be explained.

In addition, there are many indications that during anaphase there are already present two daughter spirals, at least. Electron-microscopic studies have shown that a sub-light-microscopical spiral is probably the basic element of every chromosome. If so, the known spiral should be composed of a bundle of different ultraspirals. Nevertheless, it is premature to seek a connexion between the proposed helical structure of deoxyribonucleic acid and the chromosome spiral.

Prof. H. D. Springall (University College of North Staffordshire) discussed the chemical structure of proteins and nucleic acids.

The study of an organic natural product follows the pattern: (1) isolation; (2) proof of purity; (3) estimation of molecular weight, and, if a macromolecule, molecular size and shape; (4) analysis; (5) degradation; (6) synthesis ; (7) if a macromolecule, study of the configuration of structural elements within the molecule.

The application of this sequence of studies to proteins and nucleic acids is very difficult because of their very high molecular weights, and their fragility and intractability. The methods devised were briefly discussed and the results summarized.

(1) Proteins. 'These consist fundamentally of polypeptide chains of $\alpha$-amino-acid residues (of about 19 different kinds). These chains are helical, most of them probably related to the $3 \cdot 6$ residue helix of Pauling. The helical chains are disposed in linear patterns in the fibrous proteins, and are folded into compact globular molecular shapes in the more soluble globular proteins.

The penetration of modern methods into protein structure is indicated by the analytical and degradative work which has established the complete structural framework of insulin (Sanger) and the complete synthesis of the nonapeptide amide hormones, oxytocin and vasopressin (du Vigneaud).

(2) Nucleic Acids. The building units of these are a few purine and pyrimidine $\mathrm{N}$-bases; the sugars, ribose or deoxyribose (giving rise to ribo- or deoxyribo-nucleic acids); and phosphoric acid. There are many theoretically possible modes of assembly of these diverse and polyfunctional components. The natural scheme involves the linkage $(a)$ ( $\beta$-glycosidic) of an $\mathrm{N}$-base to a sugar (to give a nucleoside), (b) (ester) of phosphoric acid to the sugar component of $a_{0}$ nucleoside (to give a nucleotide) and $(c)$ of nucleotides by phosphate diester formation via the $3^{\prime}$ - and $5^{\prime}$ sugar positions. The polynucleotide chains in deoxyribonucleic acid are arranged in twin helices about a dyad axis (Watson-Crick). The arrangement in ribonucleic acid is not known.

Synthetic work on nucleic acids is of extreme difficulty and has not yet gone beyond dinucleotides (Todd).
(3) Nucleoproteins. The general structure of these substances, especially the viruses, was briefly considered.

Prof. T. Caspersson (Stockholm) spoke on the cytochemistry of the nuclear elements.

The lecture gave a summary of the early cytochemical studies of the metabolism of the cell nucleus, mainly carried through by aid of ultra-violet ultramicrospectroscopy. The central point had been the study of the role of the polynucleotides. Especial attention was given to the conspicuous changes in the protein content of various nuclear structures during mitosis and during the interphase, the functional stage of the cell.

In the latter part of the lecture it was pointed. out that at a certain stage these studies were virtually entirely blocked by a series of technical difficulties, mainly arising when dealing with the protein components. This situation led to a comprehensive investigation aiming at development of the methods in different directions. Very recently major advances in this field have been reported and thus the cytochemical problems concerned in cell nuclear metabolism can now be attacked in a novel and hitherto inaccessible way. The technical procedures were not presented in any detail as very few data on chromosomes, gained with the new techniques, are, as yet, available. These are discussed, for example, in Cold Spring Harbor Symposia in Quantitative Biology, 1956.

Mr. N. W. Pirie (Rothamsted) dealt with the recognition, distribution, and action of nucleic acids.

There is no unequivocal way to recognize nucleic acid that has not been isolated. Methods depending on the determination of phosphorus extracted by different procedures can be confused by other phosphorus compounds; methods depending on the precipitation of the bases and determination of nitrogen are even more fallible; methods depending on the determination of ultra-violet absorption can be misleading both because of the presence of other substances with a similar absorption spectrum (for example, ascorbic acid) and also because of the effects of orientation. Feulgen staining for aldehydes is a more trustworthy method of recognizing deoxy. ribonucleic acid than any of the methods for ribonucleic acid; but here, too, there are difficulties.

The use of nuclease preparations increases the precision of these determinations; but their value depends on the demonstration, rather than the assumption, that the preparation is free from other relevant enzymes. Lack of action can be due to inaccessibility of the nucleic acid or to the presence of inhibitors. There is little evidence for the occurrence of free nucleic acid; but the nature of the linkage to proteins and other materials is obscure and probably diverse. This fact need not complicate histochemistry, because the techniques of fixation generally denature the protein and liberate any bound nucleic acid; but it complicates attempts to interpret the mode of action of nucleic acid, for the acid may be an artefact. There is some evidence for changes analogous to denaturation in nucleic acids; if such a phenomenon exists it extends still more widely the already ample possibilities for isomerism and specificity.

Deoxyribonucleic acid tends to be confined to the nucleus but is often found elsewhere, for example, egg-white and viruses, and may not have been looked for carefully. Ribonucleic acid is present nearly everywhere in organisms though it is often concentrated in regions such as microsomes and the 
bacterial cell wall. Not all deoxyribonucleic acid is present as genes : What is the status of the common generalization that all genes are deoxyribonucleic acid? A priori this seems unlikely. Similarly, regions of high metabolic activity are often rich in ribonucleic acid: Are regions of protein synthesis characteristically richer still ? It is surprising that there seems to be so little deoxyribonucleic acidribonucleic acid interconversion, and that actions such as the stimulation of polysaccharide and enzyme synthesis have been found so seldom.

Prof. A. Gustafsson (Stockholm) spoke on regularities and intentional control of mutation processes in plants.

From comparative experiments on barley, using $X$-rays, neutrons and specific chemical compounds as mutation-inducing agents, Gustafsson and his collaborators conclude that chromosome breakage and consequent $X_{1}$ sterility can be induced in barley by certain specific compounds: (1) without visible mutations of the chlorophyll apparatus, for example, 8-ethoxycaffein, (2) probably with such mutations, for example, ethylene oxide and mustards. Some agents, for example, nebularine, do not cause chromosome breakage, rearrangements, or $X_{1}$ sterility, but may increase mutation-rates considerably and cause fundamental changes in the mutation spectrum.

Chromosome breaks induced by strongly ionizing neutrons do not differ from those induced by $\mathrm{X}$-rays. Random breaks, re-attached to form translocations, rarely produce noticeable changes in morphology, anatomy or ecology, but such translocations are highly instrumental in the genetical isolation of plant populations.

Plant-breeding in its genetical aspects rests on a profound knowledge of genes and chromosomes, the importance of heterochromatin and euchromatin, chromosome number, heterozygosity, gene recombination and mutation control. Mutations, whether spontaneous or induced, are generally regarded as arising at random. Gustafsson feels that this is not, so. The mutation spectrum varies with the type of species and mutagenic agent used. The time does not seem far off whon wo shall be able to induce chromosome rearrangements and gene changes independently from one another, and control or direct the mutation process so that mutations of distinct loci, including those of high-productivity effects, are intentionally produced.

Prof. A. Müntzing (Lund) discussed chromosomes in relation to species differentiation and plant breeding.

Some species, although morphologically indistinguishable from one another, are isolated reproductively. Lewis's work on Clarkia has shown how a study of the differentiation of populations within a species can lead to an understanding of the mechanism of speciation in that group, and the part played by chromosome re-arrangements at the intra- and inter-specific levels. The salivary gland chromosomes of Drosophila have provided a means by which the nature of this chromosome differentiation can be studied in detail. In only a few cases has it been adequately demonstrated that the difference between two species is genic only and that chromosomal rearrangements have not occurred. Change in chromosome number to give an aneuploid series may result from fusion or breakage of chromosomes with diffuse centromeres.

Prof. Müntzing also spoke of the part played by polyploids in plant breeding and the value such plants had. Cytological analysis had shown the origin of many polyploid cultivated species and how they could be resynthesized, so that fresh genetic material could be incorporated into existing varieties. New polyploids have also been made and found to be of value in plants such as rye, clover, turnips and fruit trees. By the use of wheat plants lacking a pair of homologous chromosomes (nulliscomics) it has been possible to determine on which chromosome various genetic factors are situated and also to transfer whole chromosomes, bearing genes for disease resistance, to other varieties.

Prof. C. D. Darlington (Oxford) discussed the means of communication in the cell. These are to be inferred from chemical, genetical and radiation experiments and from comparative developmental studies. The co-operation and competition of nuclei had been revealed by classical experiments the meaning of which was now becoming clear in genetic and chemical terms.

The co-operation of gene activities and the co-ordination of chromosome movements both required communication along the chromosomes themselves. Evidence in relation to nucleoli, heterochromatin, and especially centromeres, showed that this communication was sometimes canalized along the chromosomes, a property which was variable and physiologically controlled. The inference of position effects from breeding experiments could thus be greatly extended. Further gene activity and integration could be seen as conditional not only on the local character of the cytoplasm but also on its eyclical changes. These cyclical changes in turn were co-ordinated in two normal systems (mitosis and meiosis) in every species. The relations of these normal systems were made clear by the abnormal systems which have now been described in many species and could be provisionally interpreted in terms of deoxyribonucleic acid and protein relations. With these cytological inferences the main concepts of genetics and embryology were consistent but they appeared in a new and larger perspective.

The author is indebted to Prof. C. D. Darlington (Oxford) and to Mr. K. M. Goodway (University College of North Staffordshire) for assistance in the preparation of this account. H. D. SpRINalal

\section{BRITISH WELDING RESEARCH ASSOCIATION}

\section{OPEN DAY AT THE ABINGTON LABORATORY}

T our present society, which is committed to the pursuit of technology in support of a material standard of life, research associations and similar bodies form an essential communication between fundamental science and industry. This theme was developed by the Minister of Supply, Mr. R. Maudling, when proposing the toast of the British Welding Research Association at the annual luncheon and open day held at the Association's laboratory at Abington, Cambridge, on July 24. The Minister went on to encourage the production of memoranda containing the essence of sound practice supported by scientific investigation, the commencement of which in the case of the Association in fields such as brazing and the construction of are-welded machinery has been made possible by application of Foreign Operations Administration funds from the United States. In 